\title{
Commentary: Book Review: Big History and the Future of Humanity
}

\author{
Fred Spier* \\ Institute for Interdisciplinary Studies, University of Amsterdam, Amsterdam, Netherlands
}

Keywords: big history, big history and the future of humanity

\section{A commentary on}

Book Review: Big History and the Future of Humanity

by Chialva, D. (2015). Front. Phys. 3:89. doi: 10.3389/fphy.2015.00089

I would like to react to the review of my book Big History and the Future of Humanity, Second Edition by Diego Chialva in this journal published on 23 November 2015, that recently came to my attention. I will address the review step by step.

Chialva:

The amount of knowledge and the diversity of the disciplines necessary to compose the wishedfor big history picture (physics, chemistry, biology, geology, history, and many more) impose a multidisciplinary approach. However, the analysis in the book is undertaken by a single author. Evidently, no single author can master the different disciplines at the required level.

\section{OPEN ACCESS}

Edited by:

Lee Samuel Finn,

Pennsylvania State University,

United States

Reviewed by:

Lowell Steven Gustafson,

Villanova University, United States

*Correspondence:

Fred Spier

f.spier@uva.n!

Specialty section:

This article was submitted to

Cosmology,

a section of the journal

Frontiers in Physics

Received: 11 April 2017 Accepted: 04 August 2017 Published: 25 August 2017

Citation:

Spier F (2017) Commentary: Book Review: Big History and the Future of Humanity. Front. Phys. 5:35. doi: 10.3389/fphy.2017.00035
Reply:

As explained in the Preface, the analysis is based on my own multi-disciplinary career that spans both the natural and social sciences over more than 40 years; on a wide reading of academic literature across all relevant disciplines; and on critical contributions by, and discussions with, a great many scholars from many different disciplines, many of them guest lecturers in our big history courses, the first of which started in 1994. Furthermore, all the chapters were read by specialists before publishing the book. All of that has involved an extraordinary amount of work. Whether I have succeeded or not will be left to the judgment of careful and knowledgeable readers.

\section{Chialva:}

The book contains several serious misconceptions about physical phenomena and their theoretical interpretations. Some of these errors are remarkably naive (for instance, on page 82 the author defines the neutrinos, I quote, "little neutrons," oblivious of a century of study in fundamental interactions), many are fundamental (for example concerning the radiation era, the role of "empty space" within atoms, and so on).

Reply:

I did not define neutrinos as little neutrons, but only indicated between brackets that this is the literal meaning of the word neutrino. This is what I wrote on p.82: "At about $10^{-4}$ seconds after the big bang, the circumstances became right for the freezing out of the much lighter leptons, most notably electrons as well as the very tiny neutrinos (little neutrons)." In the next edition I will put this between quotation marks to avoid any possible misinterpretations.

Because Chialva did not indicate in specific what went wrong with my rendering of the "radiation era, the role of 'empty space' within atoms, and so on," it is impossible for me to react to these unsubstantiated allegations. 


\section{Chialva:}

Other mistakes directly affect the pillars at the basis of the author's analysis. The author indeed claims a single principle underlying all evolution in the universe (physical, chemical, biological, cultural, and so on): the growth of complexity determined by energy flow and Goldilocks conditions. Unfortunately, his presentation of the three basic concepts (complexity, energy and Goldilocks conditions), plus a fourth extremely relevant one (entropy) is either incomplete, problematic or incorrect.

Reply:

The central thesis of the book is that the rise and demise of complexity in big history can be understood by examining energy flows through matter within certain favorable (Goldilocks) circumstances. Yet any rise of complexity somewhere inevitably leads to the rise of entropy somewhere else. Whether these terms are used correctly in my book or not will be discussed below.

\section{Chialva:}

A most evident case is energy. Let us recall that energy is a concept central to physics since about a couple of centuries. It is defined both conceptually and operationally. Rather unexpectedly -and remarkably- the author writes (Chapter 2) that he could not find a complete and clear definition of energy in any physics book. He proceeds by giving a definition (I quote it: "anything that can change matter") that is absolutely empty and lumps together the concepts of interactions, physical transformations, matter and many others (but it is difficult to judge how much the author is aware of these things). All the modern concept of energy is omitted, including aspects, like its equivalence to mass, nowadays known to the educated general public at least by hearsay.

Reply:

I find it interesting to see that Chialva readily accepts my thesis that a good definition of energy seems to be lacking. Not all physicists appear to think so. As I argued on p.46, this lack of a good definition appears to be caused at least partially by the situation that energy is such a fundamental concept that it is hard to find even more fundamental concepts that can be used to define it.

To tackle this thorny issue, I wrote on p.46: "A closer examination of the effects of energy on matter has led scholars to the profound insight that it is energy - and energy alone - that can make matter change. It makes sense, therefore, to define 'energy' as anything that can change matter, including making it more, or less, complex." This formulation includes all the interactions mentioned by Chialva. The conversion of energy into matter and vice versa is discussed in Chapter 3.

\section{Chialva:}

Complexity and Goldilocks conditions present instead quite a lack of definition. Concerning complexity, lacking a truly universal consensus, the author should at least clearly indicate what definition he adopts. He seems to do so when in Chapter 2 he claims to define complexity via "an inventory list of its mayor characteristics" (I quote), but this list is not organically presented and not all of its elements are clearly indicated. The authors analysis requires defining a measure of complexity, which would be indeed a good definition of it, but the power density he proposes (following Chaisson) is very problematic, as the authors himself often admits. In fact one wonders how he can calculate power if he claims a clear definition of energy is not available in physics.

Reply:

As mentioned in Chapter 2: when even the Santa Fe Institute, which is devoted to the study of complexity, does not know how to define it, it cannot be expected from me to be able to do so well. This subject requires more academic consideration and discussion.

In Chapter 2 my approach is explained, namely by examining both the variety of its building blocks and the interactions among them, while referring to the formulation: "It is often said that a system (I would prefer 'regime') is more complex when the whole is greater than the sum of its parts" (p.48).

It is not easy to determine and/or measure levels of complexity precisely and systematically. This remains a serious problem that has not yet been solved satisfactorily. The judgment of whether my list of these aspects is well presented or not will be left to the readers.

Regarding the issues involved in employing power densities in relation to complexity: it is not at all correct to say that I "admit" these problems. To the contrary, to my knowledge I am the first to openly tackle these problems by extensively discussing them in chapter 2. As indicated in the text: "I will explicitly not employ the concept of power density as the one and only yardstick for measuring different levels of complexity. It will only be used as an indication of the energy that is needed for complexity to emerge and continue to exist" (p.61).

As can be seen in the book, following the approach pioneered by Eric Chaisson power density calculations are entirely possible using specific forms of energy, even though a good general definition of energy may still be lacking.

\section{Chialva:}

As for Goldilocks conditions, while they should somehow be conditions at which a given "complexity" can exist, their nature largely varies and no clear concrete quantitative operational definition is provided. For example, in some parts of the book Goldilocks conditions are presented as "circumstances facilitating the stabilization of irregular matter and energy flows" (page 143, 171), but elsewhere it does not appear what are their concrete features or even just added value (for example, on page 89 one reads that Galaxies formed thanks to certain Goldilocks conditions, but "since then, Galaxies have continued to exist" with different conditions-without specifying the features of the Goldilocks ones).

Reply:

I agree that a more systematic exploration of Goldilocks circumstances is needed. Doing so across all the academic disciplines, however, might take many years, because such a project opens up an entire research agenda, as mentioned in the book. I did not want to engage in such a project before writing 
the book, because the book might never be written as a result. I opted instead to break new ground without fully exploring it, and to openly acknowledge that.

In fact, a great many interesting aspects have emerged as a result of systematically examining big history, some of which could constitute entire research agendas. About 10 years ago I tried to organize such a research project, but found that it was impossible to obtain financial support in the Netherlands because big history does not fit into any of the established academic disciplines through which these money flows occur. This is a serious problem for those of us who strive for integrated academic views of the past.

\section{Chialva:}

The misuse of the concept of entropy is another very relevant problem. In fact, its meaning in the book ranges from a quantity entering the Second Law of Thermodynamics (superficially discussed on page 53), to the, literally, "contamination (entropy) in the water supply," page 267, also 236. It is quite remarkable, in this respect, that the author proudly announces that "the Second Law of Thermodynamics plays a major role in the theoretical approach advocated here."

Reply:

It is not clear to me what my misuse of the concept of entropy would be. As explained in the book, my understanding and use of the term entropy are based on Ludwig Bolzmann's statistical approach. Surely, the dispersal of chemicals in the water supply implies an increase in entropy, seen from that point of view.

While for most, if not all natural scientists the importance of the Second Law will be obvious, for most scholars in the humanities it is not. Most of those scholars may not even be aware of this law, while they would resist attributing any importance to it for their analyses. The word "proudly" seems misplaced to me, but yes, the Second Law plays a major role in my analysis, whether scholars from the humanities like it or not.

There is, however, a serious problem, namely that the Second Law was formulated for a stable system, while the expanding universe is not. As a result, there has been a need to rethink the concept of entropy. The astrophysicists Hubert Reeves and Eric Chaisson have provided fascinating insights, mentioned in my book, yet an overall focused analysis of the Second Law and entropy throughout all of big history still seems to be lacking. This may be a frontier in physics that deserves further attention.

\section{Chialva:}

The lack consistency in the analysis is evident also in other fundamental aspects. For example, the author finds the pinnacle of the claimed overall increase of complexity of the universe in the human phenomenon, capable to create forms of artificial complexity. However, it is difficult to argue that the much lower complexity of agricultural landscapes and human settlements, compared to the much higher complexity of the natural landscapes they replaced, supports the author's conclusion about an increase in the overall universe complexity (in fact the author seems to acknowledge the problem and argue for a sort of "increased productivity [.... from a human perspective," page 237,220, in substitution of the neutral concept of complexity).

Reply:

I would never have stated that the "pinnacle of the claimed overall increase of complexity of the universe in the human phenomenon," but do interpret human societies as the greatest known forms of complexity in the known universe.

While analyzing the rise and demise of complexity, the issue at stake is to describe and explain the emergence of levels of greater complexity in relatively small pockets of the universe, while the rest of the cosmos is becoming less complex or remains more or less the same. As I argued in chapter 7, during the agricultural revolution human cultural complexity increased, while the landscapes these humans started to influence usually became less complex as a result. I never wrote that this development represented "an increase in the overall universe complexity." Such a sloppy formulation should therefore not be attributed to me in any way.

\section{Chialva:}

Overall, in particular when considering the physical evolution of the universe, the author appears to bend the data to his hypothesis. He also frequently does not give evidence for his statements, adopting expressions like "I believe," "I think."

Reply:

I would like to see specific cases in which I bend data to fit my hypotheses. Right now, this statement is no more than an unsubstantiated allegation. In fact, I made a great effort to support the analysis by a great many scholarly references, as can be seen in the bibliography and the endnotes. But in an overview of big history one cannot reference every detail in specific, because doing so would make the text unreadable.

I never used the expression "I believe" (I scanned the whole text). The only time it occurs is in a quotation from the work of US paleoanthropologist Rick Potts on p.192. The expression "I think" occurs five times in the book -so less than once per chapter-, in all cases justified, or so it seems to me.

\section{Chialva:}

Finally, while big history could have its merits as a holistic attempt to study of the universe opposed to more reductionist approaches, does the book fulfills its claimed aim of being an introduction and justification of it, showing "general patterns that would remain obscured if one were to examine only smaller portions of our past"?

The answer unfortunately is not positive: the scientific mistakes concerning concepts at the basis of the general pattern claimed by the author, make his analysis weak and generally this does not add to the results obtained by the "small histories" provided by the specific scientific, social, historical investigations. Reply:

Because Chialva's review is based on a considerable number of misrepresentations and unsubstantiated allegations, the readers may be well advised to form their own opinions concerning 
the merits of my book by reading the text themselves. The accompanying website www.bighistory.info may offer a good starting point for doing so.

\section{AUTHOR CONTRIBUTIONS}

The author confirms being the sole contributor of this work and approved it for publication.
Conflict of Interest Statement: The author declares that the research was conducted in the absence of any commercial or financial relationships that could be construed as a potential conflict of interest.

Copyright $\odot 2017$ Spier. This is an open-access article distributed under the terms of the Creative Commons Attribution License (CC BY). The use, distribution or reproduction in other forums is permitted, provided the original author(s) or licensor are credited and that the original publication in this journal is cited, in accordance with accepted academic practice. No use, distribution or reproduction is permitted which does not comply with these terms. 